\title{
EDITORIAL
}

\section{COLOMBIA MÉDICA EN EL ISI: NUEVO RETO PARA EL SIGLO XXI}

Cuando se creó Acta Médica del Valle, a comienzos de 1970, se planeó crear una revista biomédica que permaneciera a través del tiempo, por la calidad de su contenido. Y gracias a la dedicación y esfuerzo generosos de sus autores, sus editores, sus pares evaluadores, su personal de apoyo y en general de todos los que con su trabajo construyeron una revista se logró la excelencia y ahora está llegando al primer lugar de las publicaciones médicas de Colombia con el nombre de Colombia Médica (1980).

Liderados inicialmente por el doctor Rodrigo Guerrero y su grupo editorial, entre 1970 y 1972, y luego del «alumbramiento» inicial, la revista se fue estableciendo como uno de los aportes literarios científicos biomédicos más importantes en Colombia. Esta «criatura» intelectual empezó a crecer con el paso del tiempo en el número de manuscritos recibidos, aceptados y publicados, así como en su presencia y visibilidad nacional e internacional, contando para ello con la colaboración de numerosos profesionales bajo la tutela editorial de los doctores Francisco Falabella entre 1973 y 1998; Pablo Barreto, Guillermo Llanos y Francisco Falabella entre 1998 a 2001 y Guillermo Llanos desde el año 2002 hasta la fecha. Todos ellos han dejado con sus plumas, unas más gastadas que otras, su sapiencia y arduo trabajo editorial, para ayudar entre todos a editar hasta hoy 171 números de esta revista, de manera ininterrumpida, algo digno de ser tenido en cuenta en la ciencia e investigación en Colombia, por todo lo que se ha generado y producido de manera escrita y más recientemente, elaborado de manera electrónica desde 1998, siendo pionera en Colombia en las publicaciones virtuales.

Colombia Médica entró a la era cibernética desde 1998, contando a la fecha con un promedio diario de 400 visitas a su página web: http://colombiamedica. univalle.edu.co, ubicándose con el paso del tiempo entre las mejores publicaciones científicas, alcanzando la categoría A2 en el Índice Bibliográfico Nacional Publindex de Colciencias. Más aun, los esfuerzos realizados durante décadas pasadas fueron la semilla perfecta para alcanzar otros logros dentro de esta metamorfosis literaria científica al haber sido aceptada también, en diferentes e importantes bases de datos mundiales incluyendo SciELO, HINARI y ahora, Colombia Médica fue integrada en el índice de citaciones ISI (Science Citation Index-Web of Science) después de cumplir con todos los estándares de calidad, culminando así, al menos parcialmente, estos primeros 39 años de trabajo disciplinado, e iniciando un nuevo camino que llevará a Colombia Médica a una mejor posición en el escenario de las revistas médicas en el mundo.

La base de datos Thomson ISI (Institute for Scientific Information) es una institución privada, creada en la segunda mitad del siglo XX por Eugene Garfield, que entre otros requisitos exige que una revista cumpla con estándares de publicación de revistas científicas, tenga cobertura temática, posea representatividad internacional y haya un análisis de citas ${ }^{1-3}$. Este índice se especializa en el análisis de la citación, por lo cual es posible consultar cuántas veces ha sido citado cada artículo, y quién lo ha citado mediante una base de datos llamada Science Citation Index (SCI). De igual forma, ISI lista el Factor de Impacto Numérico (FIN) de cada una de las revistas que está incluida aunque discutido en diversos escenarios académicos ${ }^{1-3}$, es una de las medidas cienciométricas que ha tenido mayor auge a través de los años, para evaluar diferentes procesos académicos y científicos del mundo.

ISI ha expandido su base de datos a Latinoamérica, y a partir de julio incluyó por primera vez, 8 revistas científicas colombianas en su índice, de las cuales Colombia Médica es la única con un enfoque a personal de salud y medicina general. De esta manera, Colombia Médica y todos sus colaboradores tendrán una mayor visibilidad internacional y estarán sometidos a estos indicadores y otros aspectos relacionados que incluyen el factor de uso, el de calidad, que son parte de la revolución moderna en impactometría, cienciometría y webometría ${ }^{1-4}$ que juegan un enorme papel en determinar el reconocimiento atribuido a las publicaciones científicas. Esto es tenido en cuenta en la constitución del Sistema Nacional de Indexación de Publicaciones Especializadas de Ciencia, Tecnología e Innovación al escalafonar las revistas nacionales.

Por todo lo anterior, Colombia Médica agradece 
muy sinceramente a los 3,645 autores que han hecho sus aportes científicos a través de los años, quienes han visto coronar con éxito sus esfuerzos al publicar los resultados de sus investigaciones en la revista; gracias plenas, también, a todos los evaluadores quienes han sacado tiempo de sus ocupadas agendas, para hacer de cada trabajo evaluado una mejor obra; gracias a quienes nos apoyan, principalmente a la Universidad del Valle, de quien Colombia Médica pasó a ser parte de su patrimonio desde 1994. Finalmente, gracias a todos aquellos que, estamos seguros, seguirán apoyando este proceso lleno de nuevos retos y desafíos, en estos tiempos de comunicación prolífica, para seguir impactando cada vez más, con mayor y mejor calidad, a la comunidad científica mundial con esta publicación cuyo objetivo, a partir de la fecha, no sólo será dar frutos convencionales, sino aquellos que perduren y se renueven día a día durante el resto del presente siglo.

\section{REFERENCIAS}

1. Leon-Sarmiento FE, Bayona-Prieto J, Leon-S ME. Concepciones, confusiones y contradicciones del factor de impacto en Colombia. Rev Esp Salud Publica. 2007; 81: 147-54.

2. Leon-Sarmiento FE, Leon-S ME, Contreras VA. El impacto del factor del impacto: ¿mito o realidad? Colomb Med. 2007; 38: 290-6.

3. Garfield E. The history and meaning of the journal impact factor. JAMA. 2006; 295: 90-3.

4. Leon-Sarmiento FE, Bayona-Prieto J, Bayona E, Leon ME. Colciencias e inconciencias con los cientificos colombianos: de la edad de piedra al factor de impacto. Rev Salud Publica. 2005; 7: 227-35

Fidias E. Leon-Sarmiento, $\mathrm{MD}, \mathrm{PhD}$ Unidad de Trastornos del Movimiento Sección de Neurología Clínica Fundación Santa Fe \& Grupo Mediciencias Universidad Nacional, Bogotá, Colombia Fundación Tomodachi, Bogotá, Colombia e-mail:feleones@gmail.com 